\title{
Mapeo digital a través de la diversificación de pequeños recursos visuales para potenciar la creatividad y la autonomía de los estudiantes de primer año en el taller de proyecto
}

Experiential mapping through simple digital resources to boost creativity and empower freshmen students in the design workshop

\author{
Hernán Ascuí Fernández \\ Departamento Diseño y Teoría de la Arquitectura, \\ Universidad del Bío-Bío, Concepción, Chile \\ hascui@ubiobio.cl \\ Nelson Arias Jiménez \\ Departamento Diseño y Teoría de la Arquitectura, \\ Universidad del Bío-Bío, Concepción, Chile \\ narias@ubiobio.cl
}

\begin{abstract}
This paper expounds the teaching strategies used to introduce architecture students in the craft of design in the course Taller de Proyecto 1. These strategies are based on experiential mappings constructed from different digital resources allowing constantly confront design with real life, in order to maintain, throughout the process, a sensitive and precise relationship between reality and experience. It concludes that these methodologies strengthen the autonomy of students, developing a valuable reconnection with the way they look and transform the world, validating the premise that the act of design is an innate human act and not necessarily an erudite one.
\end{abstract}

Keywords: Experiential mapping; digital resources; teaching of architecture 


\section{Introducción}

Este trabajo plantea cómo a través de la incorporación sistemática de mapeos digitales vivenciales es posible fortalecer la autonomía del alumno durante el proceso creativo en la asignatura de Taller de Proyecto I, intensificando una exploración proyectual íntima y un apego permanente con el objeto de estudio y la realidad. La información se basa en la experiencia académica desarrollada por los autores de este artículo con un curso de 32 estudiantes, en la Escuela de Arquitectura de la Universidad del Bío-Bío en Concepción, Chile durante el primer semestre de 2016.

Una de las principales amenazas de los procesos de enseñanza-aprendizaje en los talleres de arquitectura es su alto nivel despersonalización y abstracción, lo que conlleva a una producción creativa excesivamente estandarizada, y una alarmante similitud formal en las propuestas de los estudiantes al interior de un mismo taller. Entre los factores que pueden influir en este hecho cabe mencionar la utilización no consciente y mecanizada de las herramientas de representación digital, la devoción exagerada por el culto al referente que, en muchas ocasiones, puede representar una limitación importante, tanto en la exploración formal, como en la reflexión crítica del problema a resolver; y un desmesurado protagonismo del profesor que se esmera por mantener un excesivo control formal de los resultados.

En la última década, las herramientas de diseño digitales y el desarrollo de la arquitectura paramétrica han experimentado avances sorprendentes, exhibiendo al mundo posibilidades formales insospechadas para esta arquitectura de vanguardia. Estos deslumbrantes resultados encarnan una relevante contribución para los procesos proyectuales, sin embargo, han generado también una distorsión en el rol que deberían cumplir estas herramientas en la enseñanza del proyecto. Este fenómeno ha desplazado incluso la experiencia del dibujo y otras técnicas tradicionales, algunas de las cuales, han sido responsables durante décadas de despertar habilidades transversales estrechamente vinculadas con la realidad.

En este sentido, el peligro está en que las herramientas de representación digitales contemporáneas permiten niveles de abstracción cada vez más altos, lo cual genera, con frecuencia, resultados estandarizados y desvinculados de la realidad (Gálvez, 2014), invisibilizando las sensibilidades personales de los estudiantes.
La Escuela de Arquitectura de la Universidad del Bío-Bío posee una reconocida trayectoria en la enseñanza de la arquitectura a través del cultivo de la observación de los actos humanos como fuente de conocimiento para dar origen al proyecto. Esta postura ideológica se sustenta en los postulados de Juan Borchers (1968) que se refieren al acto humano como el natural e irrenunciable punto de partida para generar nuevo conocimiento arquitectural. Esta particular manera de guiar el proceso proyectual desde la observación directa de lo que nos rodea ha generado una especialización singular que ha sido catalizada por un conjunto de académicos a través del Magister de Didáctica Proyectual MADPRO y otras iniciativas de investigación formal como URLAB y la reciente formalización del Grupo de Investigación en Didáctica Proyectual por parte de la Dirección de Investigación de la Universidad. Este sostenido trabajo ha buscado explorar metodologías emergentes asentadas en el registro de la experiencia sensoria desde una visión amplia e integradora, que permitan sistematizar experiencias significativas durante todo el proceso creativo y argumentativo del estudiante.

Este caso de estudio, desarrollado en la asignatura de Taller de proyecto I, parte de la hipótesis de que la acción de proyectar en arquitectura es un acto innato que los seres humanos poseemos como respuesta sensata, y no necesariamente erudita, ante la necesidad de generar un soporte para las actividades humanas. En sintonía con, Trigwell y Prosser (1991) se señala que para mejorar la enseñanza es imprescindible escapar de los factores que causan un aprendizaje artificial o no profundo, fragmentando los saberes bajo la estructura academicista del saber, que pone énfasis en el conocimiento acumulado por sobre el aprendizaje integral o dialógico del sujeto. Esta predisposición natural a transformar nuestro entorno, se ve claramente reflejada en los juegos de nuestros primeros años de niñez, los que activan instintivamente el pensamiento creativo para transformar la realidad produciendo un aprendizaje no solo desde la perspectiva de avances motrices, sino que logran resolver o materializar a través de recursos simples soluciones complejas que fundan las bases de nuestro bagaje proyectual íntimo, ya que están libres de todo prejuicio (figura 1).

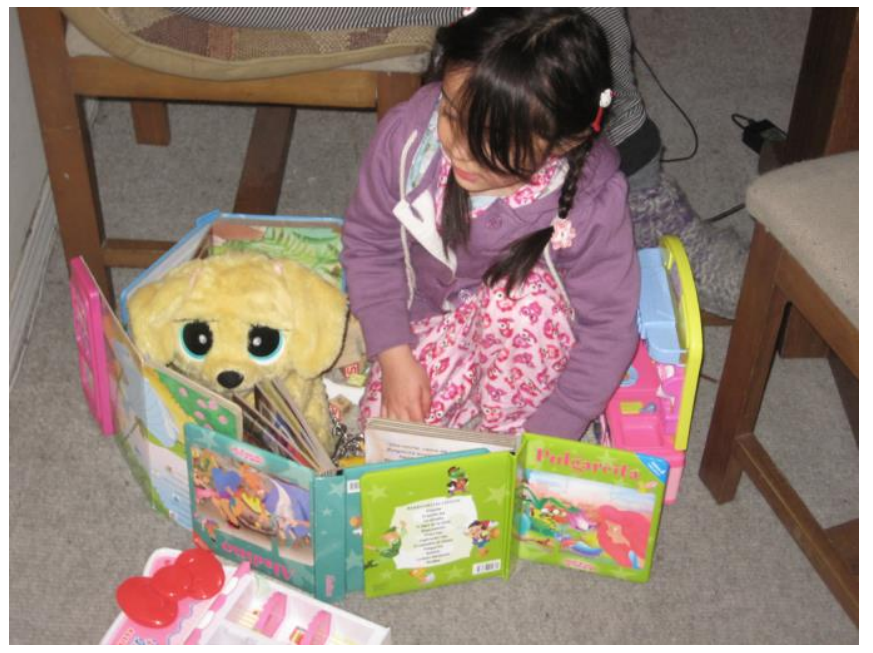

Figura 1: Experiencia cotidiana de construcción espacial lúdica de infancia. Fuente propia 
El estudiante que llega al taller para aprender a proyectar, trae consigo por lo tanto una enorme experiencia como ser creativo, proyectando espacios para jugar en su infancia, recolectando insumos disponibles a su alrededor que le permitieron plasmar su cosmovisión del mundo y del espacio a partir de su inagotable curiosidad por explorar posibilidades basadas en la lógica que regula las acciones humanas presentes en su entorno.

Si iniciamos su proceso de formación como estudiante de arquitectura desde estas prácticas constructivas lúdicas "iniciales" (ya conocidas), lo más probable es que sintonice espontáneamente con su propio imaginario y también de forma sensible y atenta con todos aquellos elementos de la realidad que puedan intervenir en el proyecto. Esto les permite mirarse a sí mismos, y hacer consciente su propia forma de comunicarse y expresarse, construyendo el conocimiento a partir de la observación directa de los seres humanos y su comportamiento; para de esta forma, ir encontrándose, lentamente con el conocimiento que la disciplina atesora en torno a las convenciones derivadas de las teorías de la forma, el espacio, la función, la estética o la física.

Para sostener este estado de conexión constante tanto con un lenguaje formal o arquitectónico propio, como con su particular manera de interactuar con los distintos ámbitos de la vida involucrados en el proyecto; se propone un Mapa Vivencial de acompañamiento, estructurado a partir de una serie de pequeños recursos visuales que va relacionando y otorgando sentido a la información recogida.

\section{Metodología}

Como Recurso didáctico para introducir a los alumnos en la acción de proyectar se realizan experiencias exploratorias de construcción de maquetas a partir de recursos ya utilizados en construcciones espontáneas comunes en los juegos de infancia, como lo son pequeñas intervenciones en la arena, y la recolección de materiales encontrados fortuitamente en la playa o en el parque. Esto les permite ir reconectando con sus habilidades como "seres proyectistas" anidadas en la memoria y desarrolladas en su niñez.
El tema del Taller de Proyecto 1 consiste en proyectar un taller de trabajo para uno mismo en un bosque al interior de la propia Universidad. Para mantener este estado creativo empoderado que se ha evocado en los ejercicios iniciales, se establece como únicos materiales válidos para elaborar las maquetas de este encargo, ramas de árboles que sean recogidas del suelo. Para poner en relación estos elementos lineales orgánicos (que luego de la recolección han sido cepilladas y/o pulidas) sólo se permite utilizar hilos encerados. Con estas restricciones metodológicas se pretende promover la definición de una lógica constructiva personal e íntima que se inicia en el momento mismo de la elección de las ramas durante los recorridos de recolección. Se pretende que la experiencia de diseñar este modelo a escala se consolide como una experiencia significativa y fuertemente sensorial desde el sentido táctil, visual e incluso del olfato. Esta aproximación, estrechamente relacionada con el mundo de lo artesanal, va generando rápidamente un lazo afectivo o de apego con el objeto, lo que refuerza esta exploración creativa íntima la que va tomando forma a partir de mecanismos que el estudiante descubre desde su propia experiencia con los nudos, empoderándolo progresivamente. La valoración de esta relación afectiva como estrategia didáctica se aborda de forma sostenida durante todo el semestre, tal como lo plantea Goethe (1993), fundador del movimiento fenomenológico, quien expone que la generación de cualquier tipo de conocimiento requiere del establecimiento de un lazo amoroso y reverente con el objeto de estudio. En esta misma dirección, se incorporan las ideas de Maturana (2013) que formula que todo acto racional está precedido por una emoción determinada, y que son precisamente las emociones las que determinan nuestros dominios de acción como seres humanos, y las reflexiones de Eissner (1998), que apuntan a contemplar una visión ampliada de los procesos cognitivos desde la afectividad.

El Mapeo vivencial surge entonces como herramienta fundamental para enhebrar y dar sentido de continuidad a los hallazgos que surgen de los distintos ejercicios vivenciales con el objetivo de cautelar una conexión sensible y atenta con aquellos aspectos de la realidad que intervienen en el proyecto. Los recursos visuales utilizados en este mapeo, actúan como vehículo de comunicación visibilizando y socializando el imaginario íntimo y oculto de cada estudiante, transformando esa visión personal del mundo en información arquitectónica transferible (Figura 2).

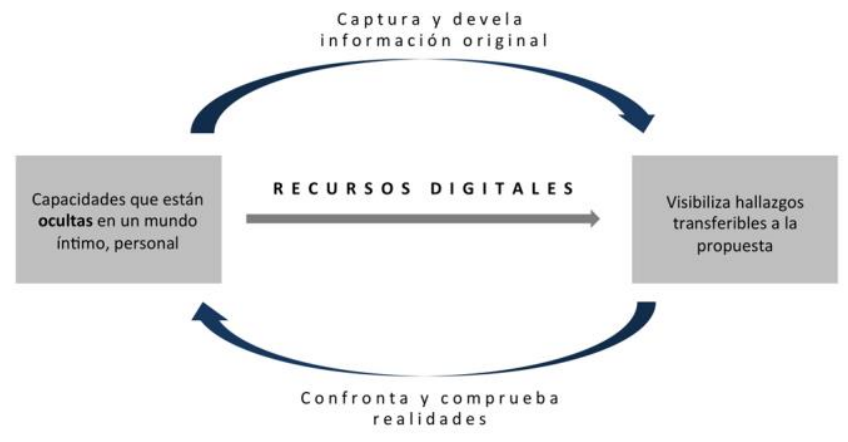

Figura 2: Esquema entorno al uso de las herramientas digitales . Fuente propia 
El Mapeo se constituye entonces como un catalizador que alimenta la conversación como experiencia consciente en torno al valor de los resultados, estimulando su interpretación y validación colectiva como comunidad de aprendizaje dialógica e intersubjetiva, tanto presencialmente al interior del Taller, como virtualmente a través de una cuenta de Twitter. Se reflexiona en torno a los valores del aprendizaje dialógico entendido a partir de la premisa de Freire (1997) de que somos seres de transformación y no de adaptación, amparando la conveniencia de las transformaciones igualitarias como resultado del diálogo. Para este efecto se incorporan una serie de prácticas comunicativas que promueven el reconocimiento y la valoración del otro, consolidando estructuras de confianza y apego que hagan posible el fenómeno social tal como lo entiende Maturana: "El amor es la emoción que constituye el dominio de conductas donde se da la operacionalidad de la aceptación del otro como un legítimo otro en la convivencia, y es ese modo de convivencia lo que connotamos cuando hablamos de lo social. Por esto digo que el amor es la emoción que funda lo social, sin aceptación del otro en la convivencia no hay fenómeno social. (Maturana, 2013:24).

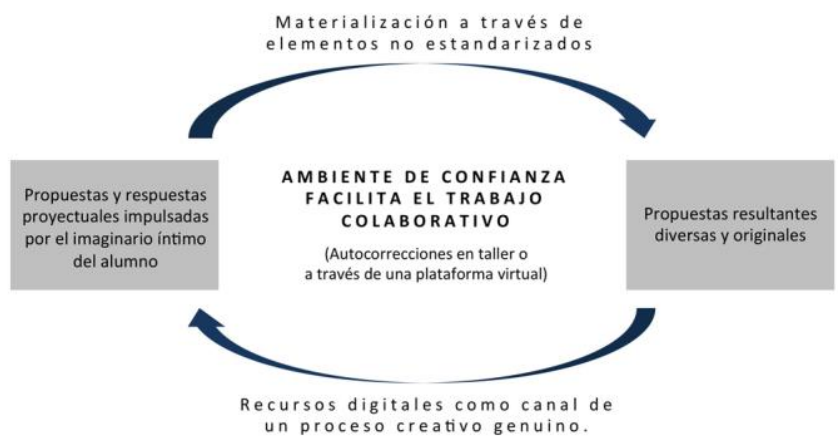

Figura 3: Esquema en torno al sentido del trabajo colaborativo. Fuente propia.

De esta forma cada nuevo producto visual o etapa de trabajo estimula al estudiante a manifestar su visión del estado de avance de un(a) compañero(a). Paralelamente, una vez por semana se desarrollan conversaciones con el grupo completo, alternando con debates de pequeños grupos a partir del método del aprendizaje basado en la colaboración y el diálogo. (Figura 3)

Apelando al autoconocimiento, se refuerza el valor de las vivencias relatadas por cada uno de los estudiantes. De esta forma, los sentidos en su totalidad (no solo la vista) son un canal de captación de información validado, transformando al estudiante en un observador libre frente a un fenómeno dinámico y no anclado a ideas preconcebidas o bien especuladas (Holl, 2011). Así, se rescata en cada uno de los ejercicios de registro, el mundo íntimo de cada uno a partir de pequeñas muestras de la realidad donde los métodos digitales no son meras herramientas de representación sino que forman "verdaderos puentes" para conectarse con el mundo. Estos ejercicios constituyen verdaderos viajes de ida y vuelta entre el pasado (memoria) el futuro (proyecto).
Se identifican los siguientes recursos digitales como hitos principales dentro del mapeo:

a-Secuencia fotográfica de registro de su propio acto de trabajar en sus talleres domésticos con su respectiva caracterización narrativa. (Figura 4 y 5)

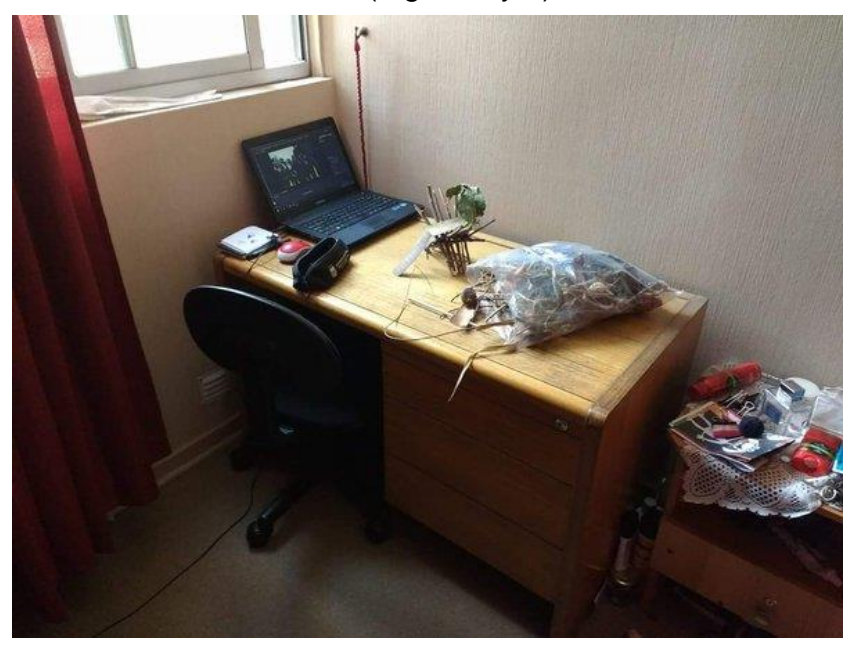

Figura 4: Espacio de trabajo propio. Fuente Juan Manuel Díaz

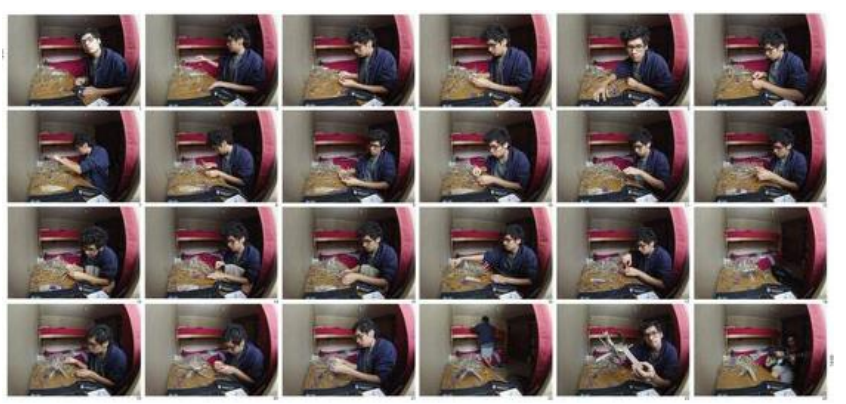

Figura 5: Secuencia fotográfica de registro del acto de trabajar. Fuente Juan Manuel Díaz

b- Ejercicio de registro in situ del lugar escogido para el proyecto en el bosque cercano a la Universidad, con acuarelas y texto narrativo vivencial en primera persona. (Figura 6)

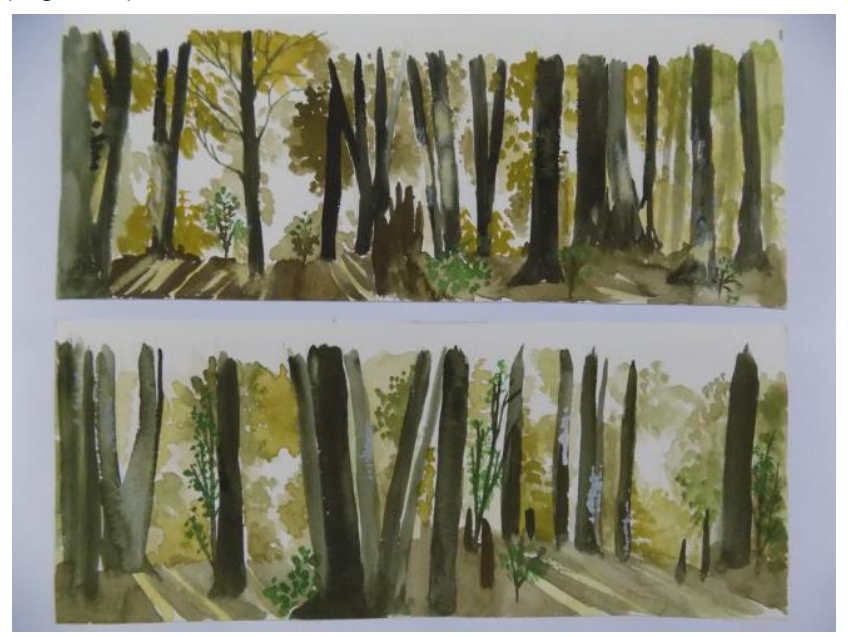

Figura 6: Ejercicio de registro in Situ con Acuarela. Fuente propia 
c-Fotomontaje del modelo resultante (maqueta) referenciado al lugar mediante el trazado de la planta del proyecto (escala 1:1) en el interior del bosque, durante un ejercicio de permanencia colectivo (Figura 7 y 8 ).

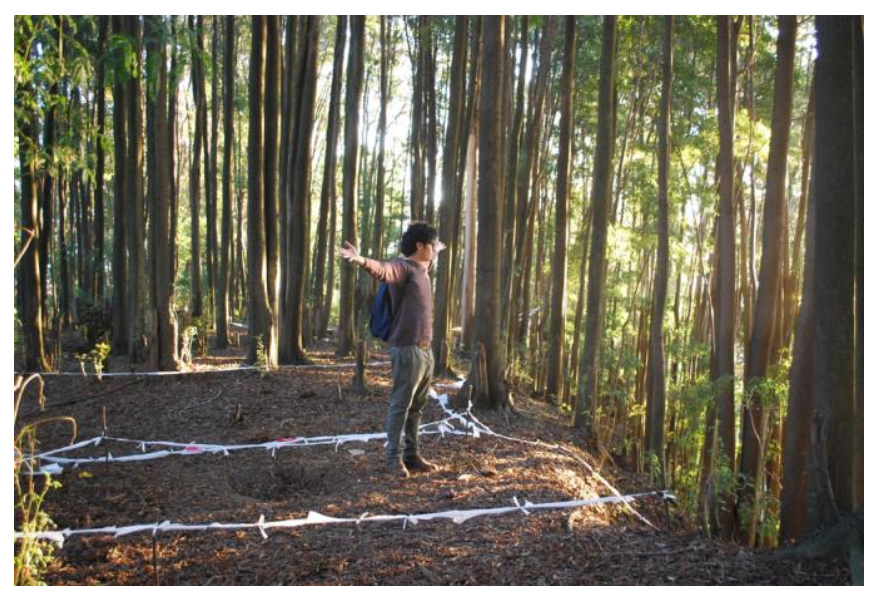

Figura 7: Demarcación in situ para fotomontaje. Fuente propia

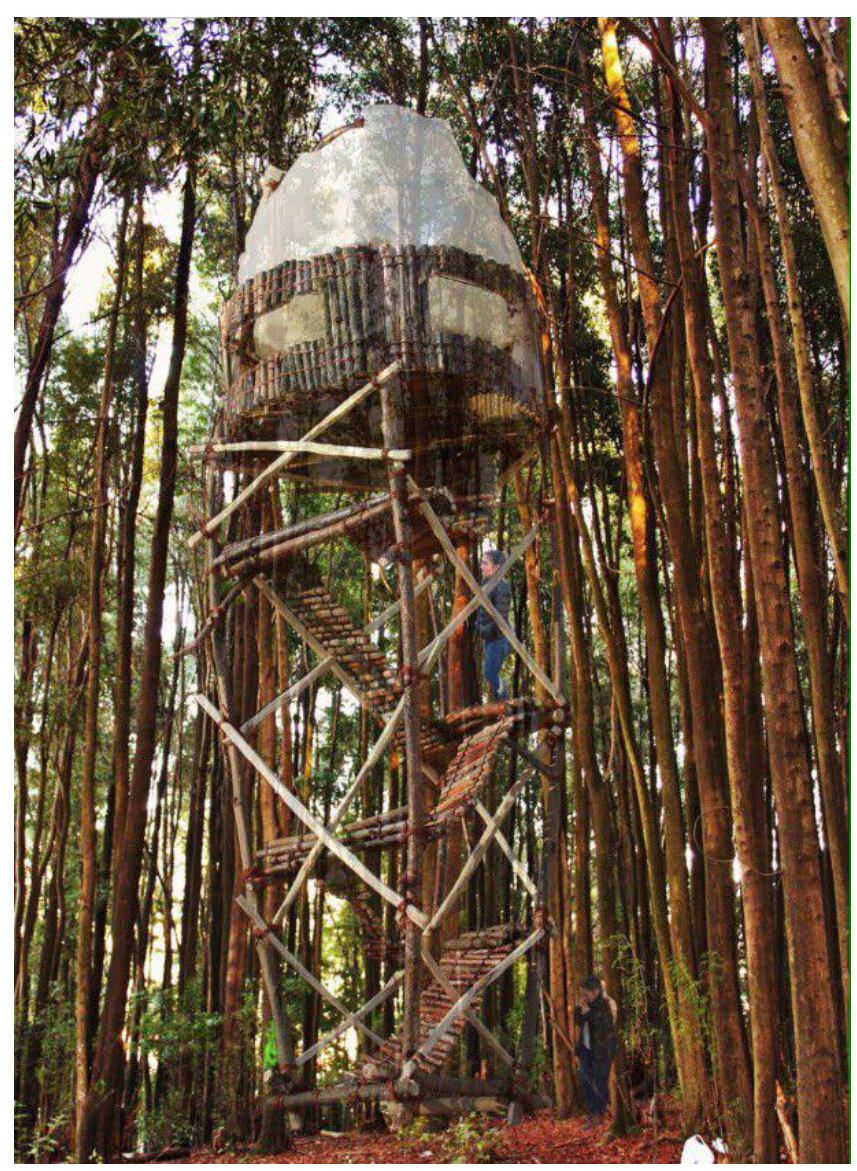

Figura 8: Ejemplo de fotomontaje, como modelo de trabajo de comprobación de la realidad. Fuente propia
Las técnicas de recolección de información para reunir la impresión de los estudiantes fueron la encuesta de preguntas abiertas, la observación directa participante y la entrevista cualitativa de grupo.

\section{Resultados}

A partir de entrevistas cualitativas de grupo, realizadas periódicamente en el taller, donde participaron todos los estudiantes junto al equipo de profesores, se logró monitorear la dinámica de aprendizaje implementada, permitiendo retroalimentación constante visualizando los siguientes resultados:

En referencia a la primera etapa que guarda relación con la secuencia fotográfica (a). Al disponer gráficamente de la sucesión de cuadros de la actividad estudiada, el estudiante reconoció en las fracciones de tiempo-espacio, las demandas del acto arquitectónico apelando a sus emociones y por consiguiente detectar el motor o el sentido de los futuros esfuerzos proyectuales.

En el ejercicio de registro in situ del lugar (b), los estudiantes lograron caracterizar, a través de la permanencia y la utilización consciente de los sentidos, el potencial del lugar, realizando un levantamiento de datos vivenciados y validados respecto al contexto de la propuesta.

Con el Fotomontaje del modelo resultante (c), los estudiantes generaron un proceso de integración, dado que confrontaron escalas (la de su modelo y la del lugar) rectificando medidas, dando paso a un ir y venir entre el lugar y el taller. Por otra parte el llevar el modelo al bosque y levantar su planta 1:1 en el terreno, les permitió corregir las perspectivas y la temperatura de las luces en la imagen digital, minimizando los niveles de abstracción y mejorando la técnica del fotomontaje para transmitir la atmósfera proyectada en sintonía efectiva con el bosque.

Por otro lado los estudiantes coinciden en que las estrategias de trabajo colaborativo fueron relevantes para generar un ambiente de interacción dinámica e intersubjetiva al interior del taller. Además les ayudó a reforzar la autoconfianza y el autoconocimiento de cada uno como seres creativos. Rescatan el uso de Twitter en cuanto a su posibilidad de proyectar el intercambio de opiniones fuera del taller, pero cuestionan su efectividad ya que al perder la posibilidad del intercambio presencial, se vuelve impersonal.

Otro canal de diagnóstico de los resultados llevado a cabo en esta investigación se realizó a través de "encuestas de preguntas abiertas", la cual consideró una muestra del 40 $\%$ del grupo de estudiantes. Se exponen a continuación extractos de las opiniones de los estudiantes referente a las experiencias citadas.

Secuencia fotográfica $(\mathrm{a})$ : 
"Es un registro importante de actos inconscientes que pasamos por alto". I "los actos desarrollados por un individuo son siempre únicos e irrepetibles" / "otorga ideas de como se posiciona y desenvuelve el cuerpo humano en el espacio" I "La secuencia fotográfica hizo percatarme de los detalles que no consideraba anteriormente como importantes en mi forma de trabajar".

Fotomontaje del modelo resultante (c):

"Verlo digitalmente en el espacio real hizo que me relacionara aún más con mi taller y ser más detallista con las dimensiones de mis espacios". / "Haber puesto el modelo en el espacio real, abre los ojos. Te hace volver a pensar en lo que nuestro cerebro descartó mientras hacíamos el modelo. Ayuda muy eficiente para afirmar la futura existencia de la obra. Un paso más a la materialización" / " Nos permite corroborar la escala real del proyecto y nuestras nociones del contexto real de lugar, la luz, el viento, la temperatura, la humedad" (Fifura 9).

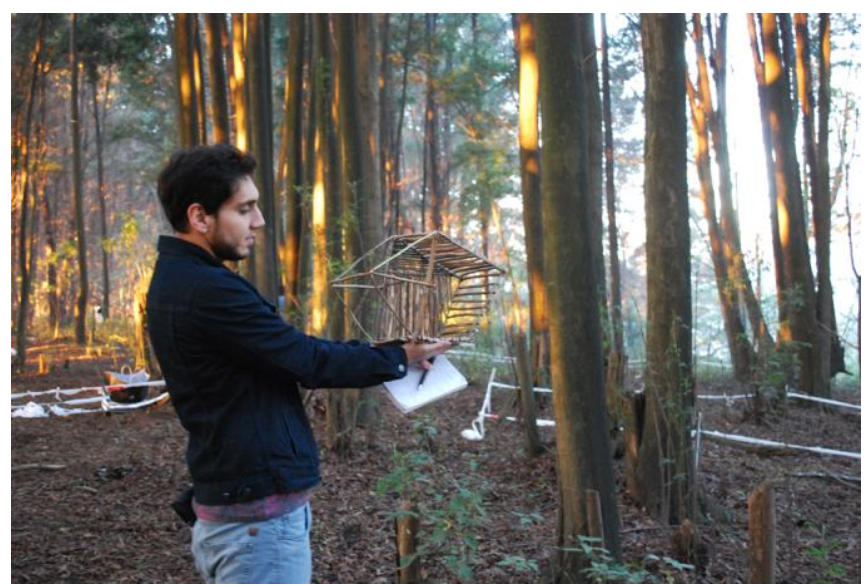

Figura 9: Ejercicios de demarcación y permanencia para fotomontaje. Fuente propia

\section{Trabajo Colaborativo:}

"Además de reforzar nuestro vínculo entre los mismos compañeros dentro del taller, se genera un espacio en el cual las ideas de todos se pueden desarrollar, cambiar, complementar y hasta ver apreciaciones que uno no vio en el proyecto, en resumen genera un crecimiento en conocimientos, experiencias y visiones" (Figura $10 \mathrm{Y} 11$ ).

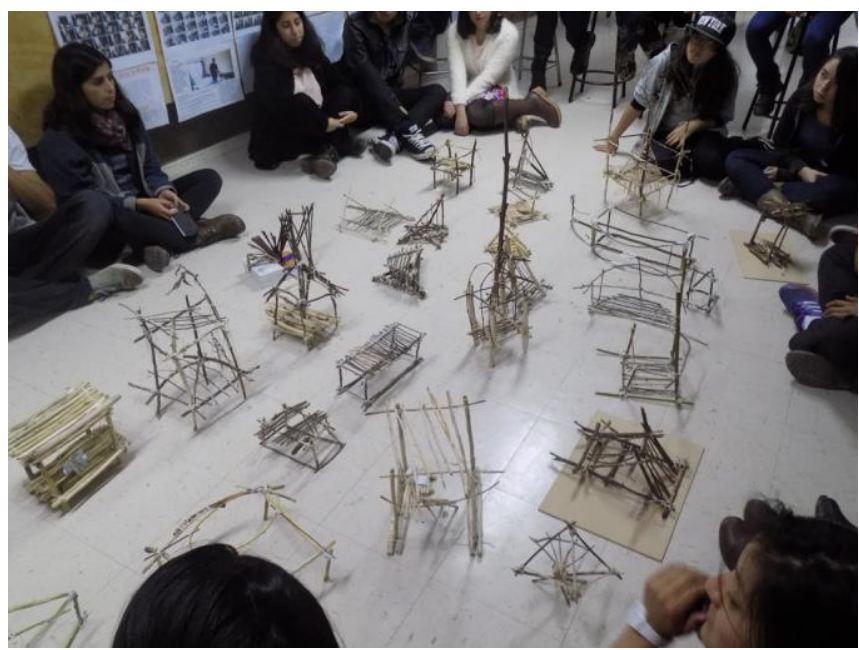

Figura 10: Sesiones de conversación en torno a los avances. Fuente propia

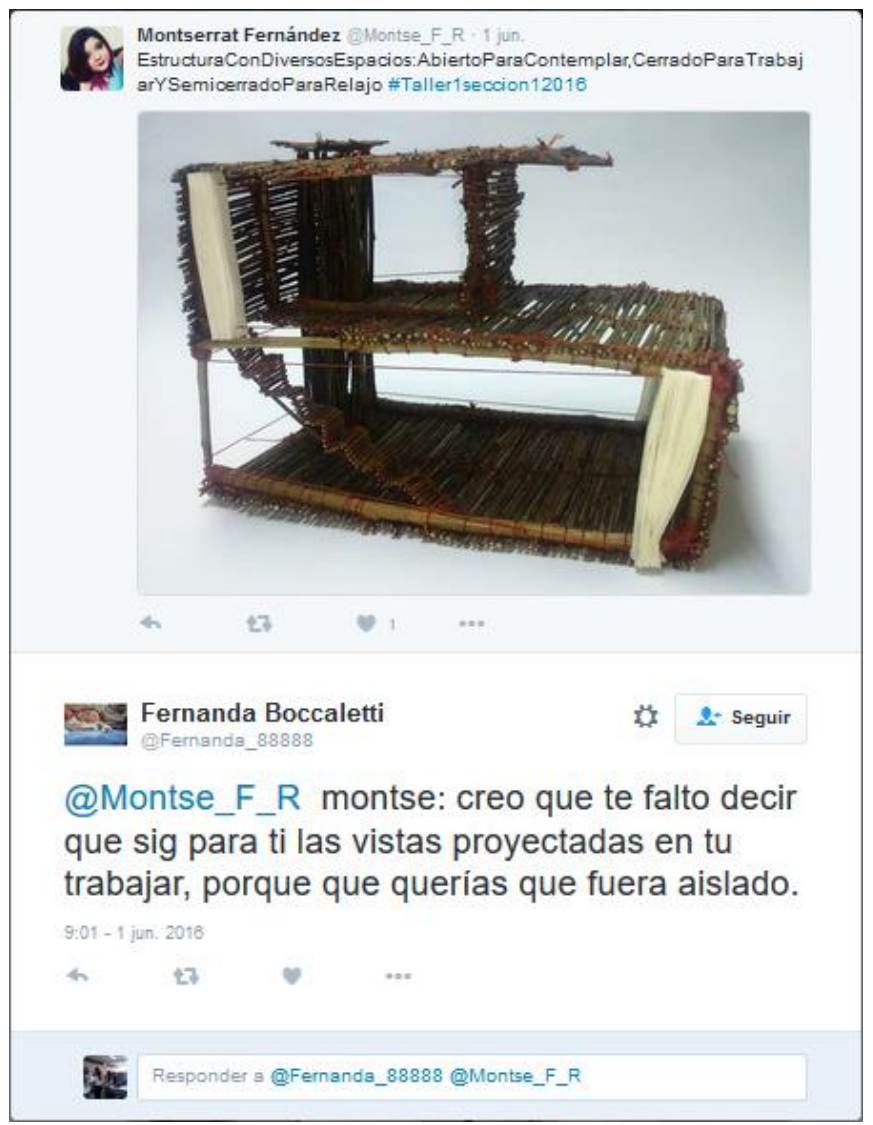

Figura 11: Sesiones de debate a través de Twitter. Fuente propia 


\section{Conclusiones}

El mapeo digital propuesto visibiliza las diferentes experiencias identificadas desde este mundo personal e íntimo, permitiendo integrar y conectar lo vivido con la propuesta. Lo anterior queda en manifiesto tras evidenciar los puntos de coincidencia entre los resultados del proceso de aprendizaje percibidos por los profesores y los estudiantes.

De esta manera se puede concluir que los recursos digitales trascienden de ser medios únicamente de representación en la enseñanza del proyecto, transformándose en potencial herramienta de registro y exploración. Esto está vinculado a la naturaleza iterativa de dichos medios, los cuales permiten levantar testimonios prístinos y desprejuiciados de los fenómenos analizados por los estudiantes, con el fin de no perder nitidez en sus parámetros de observación. Mientras que en el campo de la exploración creativa, se pudo comprobar la necesidad del cruce constante entre recursos digitales y las experiencias directas del estudiante con los fenómenos involucrados en el proyecto, a través del componente corpóreo y sensitivo.

En el marco de la investigación desarrollada por los autores que busca encontrar mecanismos efectivos para que los estudiantes de primer año inicien un proceso de revinculación con su imaginario creativo íntimo anidado en la infancia, los recursos digitales organizados a partir de mapas vivenciales se constituyen como un vehículo de comunicación sensible y eficiente que permite visibilizar y socializar los hallazgos (Figura 12).

El trabajo colaborativo como eje de desarrollo del taller permite empoderar a cada estudiante a partir de la valoración de su visión personal del mundo, el cual se valida en la experiencia reflexiva e intersubjetiva con los demás. Este espacio conversacional desarrolla relaciones afectivas basadas en la confianza y un genuino interés por el otro que potencia la diversidad y originalidad de las propuestas arquitectónicas.

Cada estudiante experimenta un sentimiento de pertenencia a esta comunidad dialógica que lo reconoce como un ser único e irremplazable empoderándolo a partir de lo que cada uno de ellos es en sí mismo y reforzando sus capacidades para conectarse racional y emocionalmente con las distintas variables que actúan en el proyecto.

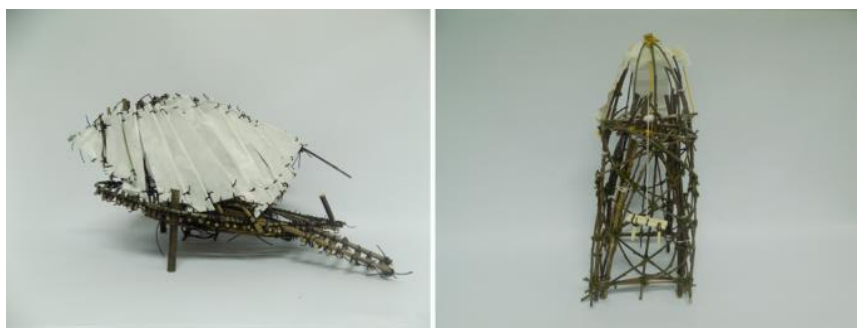

Figura 12: Ejemplos de maquetas de proyecto. Fuente propia

\section{Agradecimientos}

Se agradece especialmente a los estudiantes del curso de Proyecto I sección 1, del primer semestre de 2016 por creer en las virtudes del trabajo colaborativo y por confiar en esta propuesta medotológica. Se agradece también al ayudante, licenciado en arquitectura Eric Arriagada por su incansable contribución en esta aventura académica. Se destaca que este trabajo forma parte de los resultados del Grupo de Investigación en Didáctica Proyectual GI 160402/EF de la Universidad del Bío-Bío y se sustenta en los recursos metodológicos de la tésis doctoral "Desarrollo y evaluación de estrategias de ventilación natural basadas en la integración de torres de viento al diseño arquitectónico. Estudio de desempeño en CFD", proyecto de Investigación de Doctorado enfocado en el rescate de sistemas tradicionales (vernáculos) de mejoramiento del confort térmico, para ser aplicados en edificaciones contemporáneas.

\section{Referencias}

Borchers, J. Institución Arquitectónica. Santiago: Andrés Bello. 1968

Eissner, E. (1998). Cognición y Curriculum. Madrid: Amorrortu Editores

Freire,P. (1996). Pedagogía de la Autonomía. Río de Janeiro: Paz e Terra.

Galvez, A. (2014) El Método de proyecto análogo-digital para el mejoramiento del aprendizaje de la presentación arquitectónica dimensional., SIGraDi 2014, Procceding of the 13th Congress of the Iberoamerican Society of Digital Graphic, Montevideo, Uruguay, 12,13 y 14 de Noviembre de 2014.

Goethe, J.W.Von \& Solar, J (ed.), 1993. "Máximas y Reflexiones". Barcelona, Edhasa

Holl, S. (2011) Cuestiones de percepción. Barcelona, España. Editorial Gustavo Gili.

Maturana, H. (2013). Emociones y lenguaje. (10 Edición). Santiago: JC Sáez editor.

Trigwell, K. ;Prosser, M. (1991). Improving the quality of student learning: the influence of learning contest and student learning outcomes. Hiher Education, Volume 22, p. 251-266 